\title{
Manifestações clínicas na Policondrite Recidivante: relato de caso
}

\section{Clinical manifestations of Relapsing Polychondritis: case report}

\section{Manifestaciones clínicas en Policondritis Recurrente: relato de un caso}

\author{
Valeriana de Castro Guimarães', Gustavo Vasconcelos Nery", Fabricio Gayer M. de Araújo"', \\ Maria Alves Barbosa'v, J oão Batista Ferreirav
}

\begin{abstract}
RESUMO
A Policondrite Recidivante - PR é uma afecção sistêmica grave, de natureza imunológica, caracterizada por um processo inflamatório que acomete as estruturas cartilaginosas nasais e auriculares, vias aéreas superiores e articulações periféricas. O diagnóstico é basicamente clínico e as complicações otorrinolaringológicas podem ser as manifestações iniciais da doença. Tendo em vista a raridade da doença, objetivou-se descrever um caso de PR, atendida no Hospital das Clínicas da UFG em agosto de 2006. Para tanto, as etapas seqüenciais do atendimento foram descritas desde a consulta inicial, diagnóstico, tratamento até a alta hospitalar. As dificuldades vivenciadas pelos profissionais em dar seguimento ao tratamento, em função da resistência da paciente, provavelmente colaborou para agravamento da doença, culminando em seu óbito. O clínico deve estar atento frente a suspeita clínica de Policondrite Recidivante visando o seu diagnóstico precoce e tratamento.
\end{abstract}

Palavras chave: Policondrite Recidivante; Perda auditiva; Surdez; Doenças da traquéia; Doenças reumáticas.

\section{ABSTRACT}

The Relapsing Polychondritis - RP is a rare systemic affection, of immunological nature, characterize by an inflammatory process that affects cartilaginous structures, upper airway and peripheral articulation. The diagnosis is basically clinical and the otorhinolaryngological complications can be the initial manifestations of the illness. Having in view the rarity of the disease, it was objectified to describe a PR case attended at Hospital das Clínicas of UFG in august of 2006. Therefore, the sequential stages of the attendance had been described since the initial appointment, diagnosis, high treatment until the hospital one. The difficulties lived deeply for the professionals to proceed the treatment, in function of the resistance of the patient, probably collaborated for aggravation of the illness, culminating in its death. The physician must be alert in the presence of the clinical suspicion of Relapsing Polychondritis aiming at its precocious diagnosis and treatment.

Key words: Relapsing Polychondritis; Hearing loss; deafness; Tracheal diseases; Rheumatic diseases.

\section{RESUMEN}

La Policondrite Recidivante - PR es una afección sistémica grave, de naturaleza inmunológica. Caracterizada un proceso inflamatorio que acomete estructuras cartilaginosa nasales, vías aereas superiores y articulaciones periféricas. El diagnóstico es basicamente clínico y las complicaciones otorrinolaringológicas pueden ser las manifestaciones iniciales de la enfermedad. Esta enfermedad por ser rara, se objetivó describir un caso de PR, atendida en EI Hospital de las Clínicas de La UFG en agosto de 2006. Para tanto, las etapas secuenciales del atendimiento fueron descritas desde la consulta inicial, diagnóstico, tratamiento hasta la alta hospitalar. Las dificultades vividas por los profesionales en dar seguimiento al

\footnotetext{
Fonoaudióloga. Mestranda do Programa de Pós-Graduação em Ciências da Saúde da Faculdade de Medicina/UFG. Responsável pelo Serviço de Audiologia do Hospital das Clínicas da Universidade Federal de Goiás. E-mail: guimaraesvc@bol.com.br.

"Médico residente da Clínica de Otorrinolaringologista do Hospital das Clínicas da Faculdade de Medicina da Universidade Federal de Goiás. E-mail: gvnery@gmail.com.

III Médico da Clínica de Otorrinolaringologista do Hospital das Clínicas da Faculdade de Medicina da Universidade Federal de Goiás. E-mail: fgayer@gmail.com.

iv Enfermeira. Doutora em Enfermagem. Professora Titular da Faculdade de Enfermagem da Universidade Federal de Goiás. E-mail:maria.malves@gmail.com.

$\checkmark$ Médico otorrinolaringologista. Doutor. Pós-Doutorado em Otorrinolaringologia. Professor Adjunto do Departamento de Cirurgia da Faculdade de Medicina da Universidade Federal de Goiás. Chefe da Clínica de Otorrinolaringologista do Hospital das Clínicas da Universidade Federal de Goiás.
} 
tratamiento, en función de La resistencia de La paciente, probablemente colaboro para el agravamiento de la enfermedad, culminando en su óbito. El clínico debe estar atento frente a la sospecha clínica de Policondrite Recidivante visando su diagnóstico precoz y tratamiento.

\section{NTRODUÇÃO}

A Policondrite Recidivante - PR é uma afecção sistêmica grave, de causa desconhecida, provavelmente de natureza imunológica, caracterizada por um processo inflamatório que acomete as estruturas cartilaginosas nasais e auriculares em $80 \%$ dos casos. Ocasionalmente outras estruturas como vias aéreas superiores, articulações periféricas, olhos, vasos sangüíneos, coração e rins, podem ser afetados. A hereditariedade não contribui para o aparecimento da doença, sendo que homens e mulheres são igualmente acometidos $^{(1-7)}$.

Incomum na infância ou na adolescência, a doença surge entre 20 e 40 anos com pico de incidência na quarta década de vida ${ }^{(1,5,6)}$.

Geralmente, as manifestações iniciais de uma patologia sistêmica podem ser otorrinolaringológicas. No caso de PR os órgãos do ouvido, nariz e laringe são os mais afetados. Os episódios recorrentes de inflamação das cartilagens podem evoluir com deformidades nasais e de pavilhão auricular, além de colapso cartilaginoso laringotraqueal e hipoacusia. Freqüentemente o edema auricular pode causar estreitamento do conduto auditivo externo com obstrução da tuba auditiva, resultando em infecção da orelha média. O envolvimento do trato respiratório é tardio e raro, e quando acomete pacientes jovens, o prognóstico é bastante ruim ${ }^{(5-6)}$.

O diagnóstico da doença é basicamente clínico, sendo a biópsia das estruturas comprometidas recomendada apenas em casos em que o quadro clínico é atípico ${ }^{(1)}$. A conduta terapêutica baseia-se no uso de antiinflamatórios não hormonais, corticosteróides e imunossupressores ${ }^{(3-6,8-10)}$.

Neste artigo é proposto a apresentação de um caso, cuja relevância reside no fato deste se constituir em caso raro no ambulatório de otorrinolaringologia e em outras especialidades.
Palabras clave: Policondritis recurrente; Perda auditiva; Sordera; Enfermedades de la tráquea; Enfermedades reumáticas.

Não foram encontrados registros de atendimento de tal doença nas últimas três décadas, possivelmente pela raridade com que ocorre.

No presente relato os autores descrevem um caso de Policondrite Recidivante com manifestações otorrinolaringológicas, atendido no Hospital das Clínicas da Universidade Federal de Goiás (HC-UFG).

\section{APRESENTAÇÃO DO CASO CLÍ NI CO}

Paciente JMA, 19 anos, cor negra, estudante, solteira, natural de Aruanã - Goiás procurou o Pronto Socorro de Otorrinolaringologia do HC-UFG em agosto de 2006, com o seguinte quadro clínico: otalgia, hiperemia, edema, hipoacusia e zumbido em orelha esquerda há uma semana, sem vertigem. Relatava, ainda, ardor e vermelhidão nos olhos, dores em articulação costocondral durante a inspiração, fraqueza e falta de apetite.

Afirmou que nos últimos seis meses manifestou dois episódios de inflamação no pavilhão auricular esquerdo, procurando uma unidade de saúde em sua cidade, sendo atendida por um clínico que diagnosticou processo inflamatório prescrevendo antibióticos, com melhora do quadro após uma semana.

No momento da consulta, a paciente encontrava-se em regular estado geral, normocorada, afebril, eupneica, pressão arterial: $125 \times 80 \mathrm{mmHg}$ e pulso: $86 \mathrm{bpm}$.

$\mathrm{Na}$ avaliação clínica otorrinolaringológica a orofaringe e rinofaringe anterior não apresentavam alterações. À otoscopia, a orelha direita apresentava-se normal enquanto na orelha esquerda haviam sinais de reação inflamatória em toda a área condral do pavilhão auricular e conduto auditivo externo, sem características de infecção bacteriana. A membrana timpânica estava íntegra, mas foi observada presença de hiperemia conjuntival e 
dor moderada à palpação nas articulações costocondrais.

Diante do quadro clínico a paciente foi internada, sendo solicitado o parecer da equipe de reumatologia. Nessa ocasião, os exames laboratoriais mostraram: VHS - $71 \mathrm{~mm}$; eletroforese de proteínas, FAN, C-ANCA, CélulasLE, Fator Reumatóide, VDRL e hemograma normais.
Os

procedimentos

nasofaringolaringoscopia

e

Tomografia computadorizada de ossos temporais não revelaram alterações, já a audiometria tonal detectou perda auditiva mista descendente à esquerda com limiares auditivos dentro dos padrões de normalidade à direita conforme ilustrado na Figura 1.

\section{ORELHA DIRETTA}

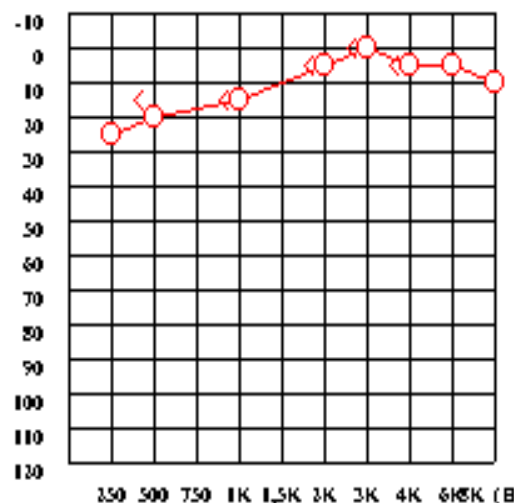

\section{ORELHA ESQUERDA}

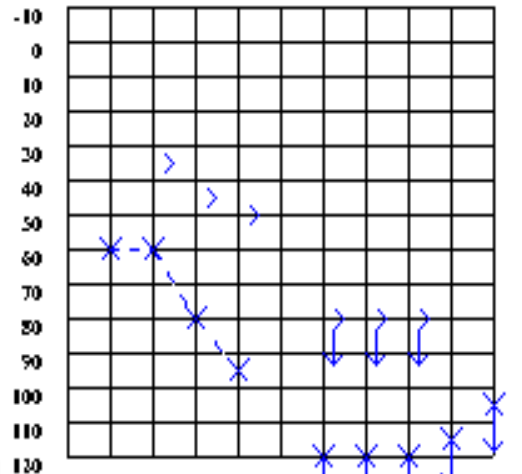

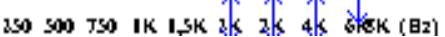

Figura 1: Audiometria tonal realizada no Serviço de Audiologia HCUFG/2006

Com diagnóstico clínico de PR, iniciou-se tratamento medicamentoso com uso de corticóide $60 \mathrm{mg} /$ dia de Prednisona e $100 \mathrm{mg} / \mathrm{dia}$ de Azatioprina, resultando melhora acentuada dos sinais e sintomas apresentados inicialmente pela paciente.

Onze dias depois da consulta inicial, a paciente recusou-se a permanecer internada, interrompendo o tratamento, sendo frustradas todas as tentativas em mantê-la hospitalizada. Retornando à cidade de origem com prescrição médica de 60mg/dia de Prednisona e $100 \mathrm{mg} /$ dia de Azatioprina com retorno posterior agendado ao ambulatório de otorrinolaringologia e reumatologia do hospital para seguimento ao tratamento.

Em decorrência da falta de comparecimento da paciente ao retorno do ambulatório de otorrino, foi realizado contato telefônico da pesquisadora com os familiares da mesma, sendo informada pela irmã sobre o óbito da paciente que havia ocorrido três dias antes. Segundo a informante, o falecimento se deu por prováveis complicações respiratórias, informações estas fornecidas pelo clínico que a socorreu em sua cidade.

\section{SCUSSÃO}

O interesse na descrição deste caso é evidenciado na raridade com que a doença ocorre nesta faixa etária, assim como o desenrolar e desfecho do mesmo.

O diagnóstico da PR é predominantemente clínico, McAdam et $a^{(11)}$ estabelece como critérios de diagnóstico da PR a presença de três ou mais dos seguintes sintomas: condrite auricular bilateral; poliartrite inflamatória não erosiva soronegativo; condrite nasal; inflamação ocular; condrite do trato respiratório; disfunção coclear e/ou vestibular e confirmação de quadro histológico por biópsia de cartilagem. No caso descrito, a paciente apresentou condrite auricular e surdez unilateral, zumbido, episclerite e esclerite, além de artrite costocondral, sugerindo clinicamente o diagnóstico da doença, não sendo realizado biópsia de cartilagem como preconizam alguns estudos $^{(1,6,11)}$.

Exames como FAN, Células LE, C-ANCA ou p-ANCA podem auxiliar no diagnóstico diferencial de outras doenças sistêmicas como: desmatopolimiosite, lúpus eritematoso sistêmico, síndrome de Cogan, doença de Behçet e artrite reumatoide e granulomatose de 
Wegner ${ }^{(2-3,7,12)}$. Como sugerido na literatura, exames laboratoriais foram realizados na paciente, sendo que apenas o VHS foi positivo, confirmando o processo inflamatório crônico compatível com PR. De acordo com McAdam et al $^{(11)}$ a condrite auricular e a perda auditiva surgem de forma bilateral, contrariando tais critérios no caso relatado houve comprometimento auditivo em uma das orelhas e condrite auricular unilateral, achados semelhantes foram descritos também por Cavalcanti et $\mathrm{al}^{(1)}$. A presença de edema no conduto auditivo externo justifica o componente condutivo da perda auditiva do tipo mista.

Apesar da paciente não ter prosseguido com o tratamento, sabe-se que a sobrevida da doença em 5 anos é de $74 \%$ e que o óbito ocorre principalmente por complicações respiratórias com comprometimento das estruturas laríngeas, traqueal ou valvular cardíaca $^{(5-6)}$. Semelhante ao caso apresentado por Cavalcanti et al $^{(1)}$ as complicações respiratórias ocorreram tardiamente, porém com agravos no quadro da paciente.

Embora sem comprovações de autópsia, as informações prestadas pela família sobre a morte da paciente ocorrido por possível complicação respiratória, estão condizentes com relatos da literatura ${ }^{(5-6)}$.

As condutas terapêuticas adotadas precocemente foram determinantes para a pronta recuperação dos pacientes como relatam alguns estudos ${ }^{(1,7,12)}$, contrário ao caso aqui apresentado em que às condutas terapêuticas não foram seguidas em função da resistência da paciente em aderir ao tratamento.

Mesmo com o diagnóstico ocorrido em tempo hábil e a conduta terapêutica estabelecida, a não adesão ao tratamento culminou com a morte da paciente. Entretanto, casos descritos na literatura apontam que a colaboração, conscientização e participação dos pacientes frente a enfermidade foram decisivos no prognóstico, demonstrando êxito mesmo diante da severidade e gravidade da doença $^{(1,7,12)}$.

\section{CONCLUSÃO}

A incompreensão e falta de colaboração da paciente e de familiares frente à doença, possivelmente pela falta de conscientização da gravidade do problema, apesar de alertados pelas equipes que acompanhavam o caso, corroborou para o lamentavelmente episódio ocorrido.

Diante da severidade, a suspeita clínica de Policondrite Recidivante deve ser considerada visando o seu diagnóstico precoce e tratamento, com apoio clínico e reumatológico, face às suas características complexas tais como autoimunidade e o curso com graves complicações que podem levar ao óbito.

Ao clínico resta ficar atento aos sinais e sintomas apresentados pelos pacientes ao procurá-lo, pois uma doença sistêmica grave como esta pode passar despercebida, comprometendo seriamente a saúde do paciente.

\section{REFERÊNCIAS}

1. Cavalcanti A, Barbosa CMPL, Len CA, Terreri MTRA, Hilário MOE. Policondrite recidivante na infância: relato de caso e revisão da literatura. Rev. Bras. Reumatol. 2007;47(3):223-7.

2. Pereira DB, Amaral JLA, Szajubok JCM, Lima SMA, Chahade WH. Manifestações otorrinolaringológicas nas doenças reumáticas auto-imunes. Rev. Bras. Reumatol. 2006; 46(2): 118-25.

3. Neves FS, Gonçalves DP, Lage LV, Gonçalves CR. Síndrome de Behçet e policondrite recidivante: Síndrome MAGIC. Rev. Bras. Reumatol. 2006;46(2): 157-60.

4. Rho YH, Choi SJ, Choi YS, Lee YH, Ji JD, Song GG. Relapsing polychondritis with aortitis without valvular involvement. J Rheumatol. 2005; 32(5): 954-6.

5. Gergely $P$ J r, Poor G. Relapsing polychondritis. Best Pract Res Clin Rheumatol. 2004; 18(5): 723-38.

6. Kent PD, Michet CJ Jr, Luthra HS. Relapsing polychondritis. Curr Opin Rheumatol. 2004; 16(1): 56-61.

7. Rodrigues EM, Roberta C. N. Silveira RCN, Leite N, Tepedino MM. Policondrite recidivante: relato de caso. Rev. Bras. Otorrinolaringologia. 2003; 69(1):128-30.

8. Carter JD. Treatment of relapsing polychondritis with a TNF antagonist. J Rheumatol. 2005; 32(7): 1413.

9. Cazabon S, Over K, Butcher J. The successful use of infliximab in resistant relapsing 
polychondritis and associated scleritis. Eye. $2005 ; 19(2): 222-4$.

10. Richez C, Dumoulin C, Coutouly X, Schaeverbeke T. Successful treatment of relapsing polychondritis with infliximab. Clin Exp Rheumatol. 2004;22(5):629-31.

11. McAdam L, O'Hanlan MA, Bluestone $R$, Peterson CM. Relapsing polychondritis: prospective study of 23 patients and a review of the literature. Medicine (Baltimore). 1976; 55(3): 193-215.

12. Schmidt L, Burkiewicz CJC, Silva MB, Skare TL. Esclerite como manifestação inicial de granulomatose de Wegener: descrição de caso. Rev Bras Reumatol. 2007;47(2): 145-7.

Artigo recebido em 30.04.07

Aprovado para publicação em 30.09.08 